\title{
Dose-related histopathology and bone remodeling characteristics of the knee articular cartilage and subchondral bone induced by glucocorticoids in rats
}

\author{
YAN CHEN, LIAN-FANG HUANG and JUE-XIN ZHU
}

\author{
Guangdong Key Laboratory for Research and Development of Natural Drugs, \\ Guangdong Medical University, Zhanjiang, Guangdong 524023, P.R. China
}

Received April 16, 2018; Accepted November 14, 2018

DOI: $10.3892 /$ etm.2019.7508

\begin{abstract}
The aim of the current study was to investigate histopathological changes and bone remodeling in the knee articular cartilage and subchondral bone in rats following treatment with glucocorticoids. A total of 30 3-month-old female Sprague-Dawley rats were randomly divided into either a vehicle control group or one of three experimental groups wherein dexamethasone (Dex) was administered at a dose of $1.0,2.5$ or $5.0 \mathrm{mg} / \mathrm{kg}$ (Dex1.0, Dex2.5 and Dex5.0, respectively), for 8 weeks. Articular cartilage and the epiphyseal subchondral bone of the proximal tibias were evaluated by histopathology or for bone remodeling using histomorphometry. No histological changes were identified in the knee articular cartilage but the bone formation rate of the subchondral bone was lower in the Dex1.0 group compared with that of the control group. Compared with the control and the Dex1.0 group, the width of the articular cartilage and the subchondral plate were larger, with abnormal morphology and increased apoptosis of chondrocytes, decreased cell/matrix volume ratio in the cartilage and fewer blood vessels in the subchondral plate in the Dex2.5 and Dex5.0 groups. A higher Dex dose resulted in more severe inhibition of bone formation, a greater number of apoptotic osteocytes and constrained bone resorption. All microstructure parameters indicated no significant changes in the Dex2.5 group but exhibited deterioration in the Dex5.0 group compared with the normal and Dex1.0 group. There were no significant differences in morphological changes, or in static and dynamic bone indices between the Dex2.5 and Dex5.0 groups. In conclusion, long-term glucocorticoid use
\end{abstract}

Correspondence to: Professor Yan Chen, Guangdong Key Laboratory for Research and Development of Natural Drugs, Guangdong Medical University, 2 Wenming Dong Road, Zhanjiang, Guangdong 524023, P.R. China

E-mail: chenyan8182@163.com

Key words: glucocorticoid, articular cartilage, subchondral bone, knee, rat induced dose-related histopathological changes in the knee articular cartilage, along with unbalanced bone remodeling and osteopenia in the subchondral bone. The degree of damage to the articular cartilage was milder and transformed from compensation to degeneration at higher doses.

\section{Introduction}

Prolonged glucocorticoid administration causes two common and irreversible skeletal problems, namely glucocorticoid-induced osteoporosis and osteonecrosis of the femoral head (1). Osteonecrosis of the femoral head, which is characterized by degeneration and destruction, involves both the articular cartilage and the subchondral bone. However, the area most vulnerable to steroid use remains uncertain. It is unclear whether articular cartilage and subchondral bone of the knee joint are also affected by excessive glucocorticoid use, in addition to the femoral head. In patients receiving glucocorticoid therapy, the incidence of osteonecrosis ranges from $9-40 \%$ and is reportedly increasing $(2,3)$. Long-term low-dose use or short-term exposure to large doses of glucocorticoids can potentially lead to osteonecrosis of the femoral head even in the absence of osteoporosis (4). Published reports suggest that osteonecrosis of the femoral head can occur as early as 36 days after steroid use even though, in most cases, osteoporosis often appears after 3 months of glucocorticoid use (5). Thus, it appears that osteonecrosis develops prior to osteoporosis and that pathological changes occur before osteonecrosis symptoms manifest themselves (6). It is known that pathological changes during osteonecrosis of the femoral head include reduced subchondral trabecular bone mass, deteriorated trabecular microstructure, thinning and degenerating of the articular cartilage, and eventual collapse of the femoral head and joint dysfunction $(7,8)$. The knee joint is similar in structure to the articular cartilage and the subchondral bone. Nevertheless, few reports have described the histopathological and bone remodeling changes in the cartilage and the subchondral bone of the knee.

Therefore, the aim of the current study was to investigate the changes in morphology and microstructure of the articular cartilage and to assess bone remodeling of the subchondral trabecular bone in the proximal tibia due to prolonged 
glucocorticoid treatment at three different doses using an experimental rat model.

\section{Materials and methods}

Animal model and grouping. The current study was performed according to the guidelines for the Care and Use of Laboratory Animals of the Guangdong Laboratory Animal Monitoring Institute (Guangzhou, China) and were approved by the Academic Committee on the Ethics of Animal Experiments of Guangdong Medical University (Zhanjiang, China) with permit number SYXK (Guangdong) A2008036. A total of 30 female Sprague-Dawley rats (age, 3 months; weight, 180-200 g) were provided by the animal center at Guangdong Medical University (Zhanjiang, China). All rats were housed under identical conditions with controlled temperature $\left(24-28^{\circ} \mathrm{C}\right)$, relative humidity (50-70\%) and 12-h light/dark cycle with free access to fresh water and a normal diet.

All rats were divided into the following four groups (mean body weight, 180-200 g): Vehicle control (Cont, saline alone, $\mathrm{n}=7$ ) or dexamethasone (Dex) sodium phosphate (provided by the College of Pharmacy, University of Nebraska, Omaha, NE, USA) at doses of 1.0 (Dex1.0, $n=7), 2.5($ Dex 2.5, $n=8)$, and $5.0 \mathrm{mg} / \mathrm{kg}$ (Dex5.0, $\mathrm{n}=8$ ). Dex sodium phosphate was dissolved in saline and was injected intramuscularly $(1 \mathrm{ml} / \mathrm{kg})$ twice/week for 8 weeks. The Dex dose and administration route were selected based on published literature (9).

Histopathology and evaluation of bone remodeling characteristics. At the end of the experiment, all tibias were harvested. Before subjecting them to gradient dehydration and methyl methacrylate resin embedding, the proximal tibia with an intact lateral plateau of articular cartilage was stripped of soft tissues and sawed on the frontal plane using an IsoMet precision bone saw (Buehler, Lake Bluff, IL, USA) to expose the marrow cavity. Next, undecalcified bone plastic blocks were sliced at thicknesses of 9 and $4 \mu \mathrm{m}$ on a hard tissue microtome (Leica RM2255, Leica Microsystems GmbH, Wetzlar, Germany). The unstained $9-\mu \mathrm{m}$ thick sections were directly processed for histomorphometry, while the $4 \mu \mathrm{m}$ sections were stained using the Masson-Goldner trichrome staining technique (10) (CAS Hematoxylin 517-28-2, Ponceau 2R 3761-53-3, Acid Fuchsin 3244-88-0, Orange G 1936-15-8, Light green SF yellowish 5141-20-8; Sigma-Aldrich; Merck KGaA). Sections were analyzed on a graphics tablet (DTZ-2100D, Wacom Co., Ltd., Kazo, Japan) connected to a semi-automatic digitizing image analysis system using the DP72 Olypus camera and a light microscope (BX43; Olympus Corporation, Tokyo, Japan) with a measurement software program (OsteoMeasure 3.1, Osteometrics, Inc., Decatur, GA, USA).

The thickness of the articular cartilage, including the superficial, transitional and radial zones, as well as the thickness of the subchondral zone, including the calcified zone and the subchondral cortical plate, were measured. Additionally, chondrocyte volume, size, shape and arrangement and blood vessel number were recorded and compared among the Dex groups and controls. Histomorphometric parameters in the subchondral trabecular bone area between the proximal tibial growth cartilage and the subchondral cortical plate were also analyzed. The abbreviations of the parameters used follow the recommendations of the Histomorphometric Nomenclature Committee and have been used in previous studies (11). The microarchitecture parameters analyzed were trabecular thickness (Tb.Th), trabecular number (Tb.N) and trabecular spacing (Tb.Sp). Dynamic measurement parameters, ratio of mineralizing surface to bone surface (MS/BS), bone formation rate per unit of bone surface $(\mathrm{BFR} / \mathrm{BS})$ and bone formation rate per unit of bone volume $(\mathrm{BFR} / \mathrm{BV})$ were analyzed under ultraviolet light. The number of osteoblast surfaces per bone surface (Ob.S/BS), osteoclast surfaces per bone surface (Oc.S/BS) and osteocyte lacuna volume (Osteocyte.V/BV) were also measured.

Fluorochrome labeling analysis. For double labeling in vivo, rats were administered a subcutaneous injection of tetracycline (40 mg/kg, Sigma-Aldrich; Merck KGaA, Darmstadt, Germany) on days 13 and 14 , and calcein $(10 \mathrm{mg} / \mathrm{kg}$, Sigma-Aldrich; Merck KGaA) on days 3 and 4 prior to sacrifice. Rats were euthanized by intraperitoneal injection of sodium pentobarbital $(1.2 \mathrm{mg} / \mathrm{kg})$ and $8-10 \mathrm{ml}$ blood was obtained by cardiac puncture. The left proximal tibias were harvested, as described above. Fluorescence was visualized using a fluorescence microscope (magnification, x10; Olympus Corporation).

Statistical analysis. Data are presented as the mean \pm standard deviation. The statistical differences among groups were evaluated by analysis of variance with Fisher's least significant difference test using SPSS 17.0 software (SPSS, Inc., Chicago, IL, USA). $\mathrm{P}<0.05$ was considered to indicate a statistically significant difference.

\section{Results}

Representative images indicating the thickness and morphology of the articular cartilage and the subchondral zone are presented in Fig. 1 and quantification of the associated parameters is presented in Fig. 2. There were no obvious differences between the control and Dex1.0 groups. When compared with the control and Dex1.0 groups, the total width of the articular cartilage was significantly higher in the Dex2.5 group due to greater thickness of the transitional and radial zones. Similarly, the width of the entire articular cartilage had significantly expanded in the Dex5.0 group when compared with the control and Dex1.0 groups and is attributable to the thickening of the radial and transitional zone, which countered a reduction in the superficial zone (Fig. 2A). Chondrocytes within the articular cartilage were either spherical or elliptical in shape in the normal and the Dex1.0 groups with uniform and rich cytoplasm. In contrast, in the Dex2.5 and Dex5.0 groups, chondrocytes were triangular or spindle-like with denser cytoplasm and apoptotic chondrocytes (Fig. 1), and demonstrated a significantly lower cell/matrix volume ratio in the articular cartilage (Fig. 2B). The width of the subchondral zone, including the calcified zone and the subchondral cortical plate was larger, but the blood vessel number within the cortical plate was lower in the Dex2.5 and Dex5.0 groups compared with the Dex1.0 and control group (Fig. 2C and D).

Representative images of subchondral trabecular morphology are presented in Fig. 3. Related parameters are 
A

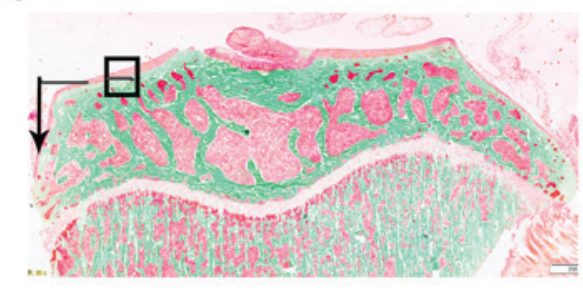

C

Cont

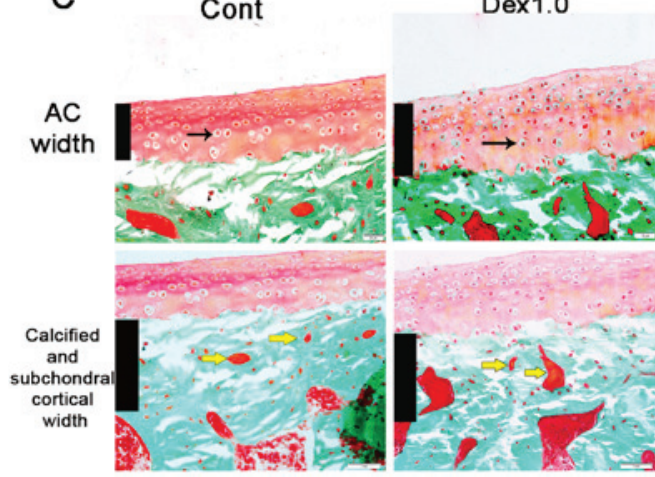

B

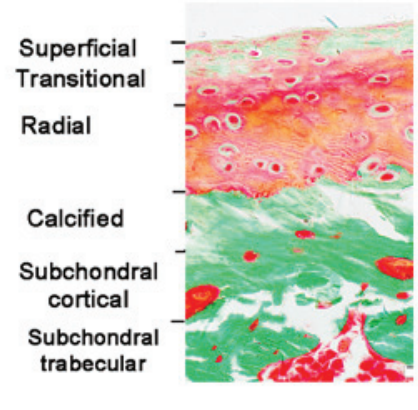

Dex2.5

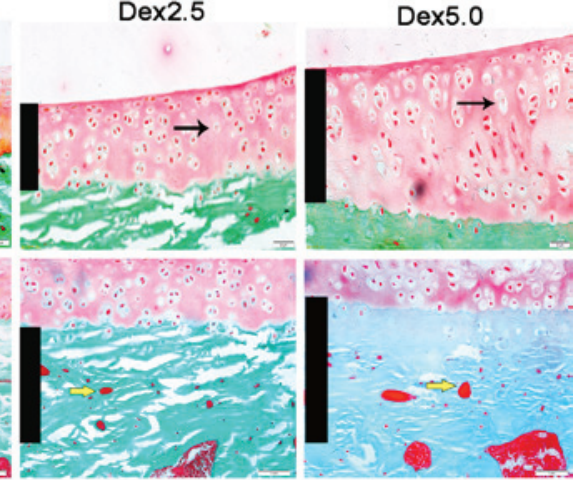

Figure 1. Morphology of the AC and subchondral zone from rats treated with vehicle or three doses of Dex. Staining was performed using the Masson-Goldner trichrome bone stain technique. (A) Image of the proximal tibial epiphyseal area with an intact lateral plateau of AC. Magnification, $\mathrm{x}$ 4. The black frame and corner arrow in indicate the observed and measured site of AC and subchondral zone, which is magnified and shown separately in panel C. (B) Enlarged section indicating the AC layers and subchondral zone shown in panel C. Original magnification, $\mathrm{x} 40$. (C) AC and subchondral zone in each group. Black columns indicate the width of either AC or subchondral zone. Black arrows point to chondrocytes in the AC. Chondrocytes are normal in the Cont and Dex 1.0 mg groups but are triangular or spindle-like with large vacuoles in the Dex2.5 and Dex5.0 groups. Yellow arrows point to blood vessels within the subchondral cortical plate. Magnification, x20. Dex, dexamethasone; Cont, control group, Dex1.0, Dex 1.0 mg/kg group; Dex2.5, Dex 2.5 mg/kg group; Dex5.0, Dex 5.0 mg/kg group; AC, articular cartilage.
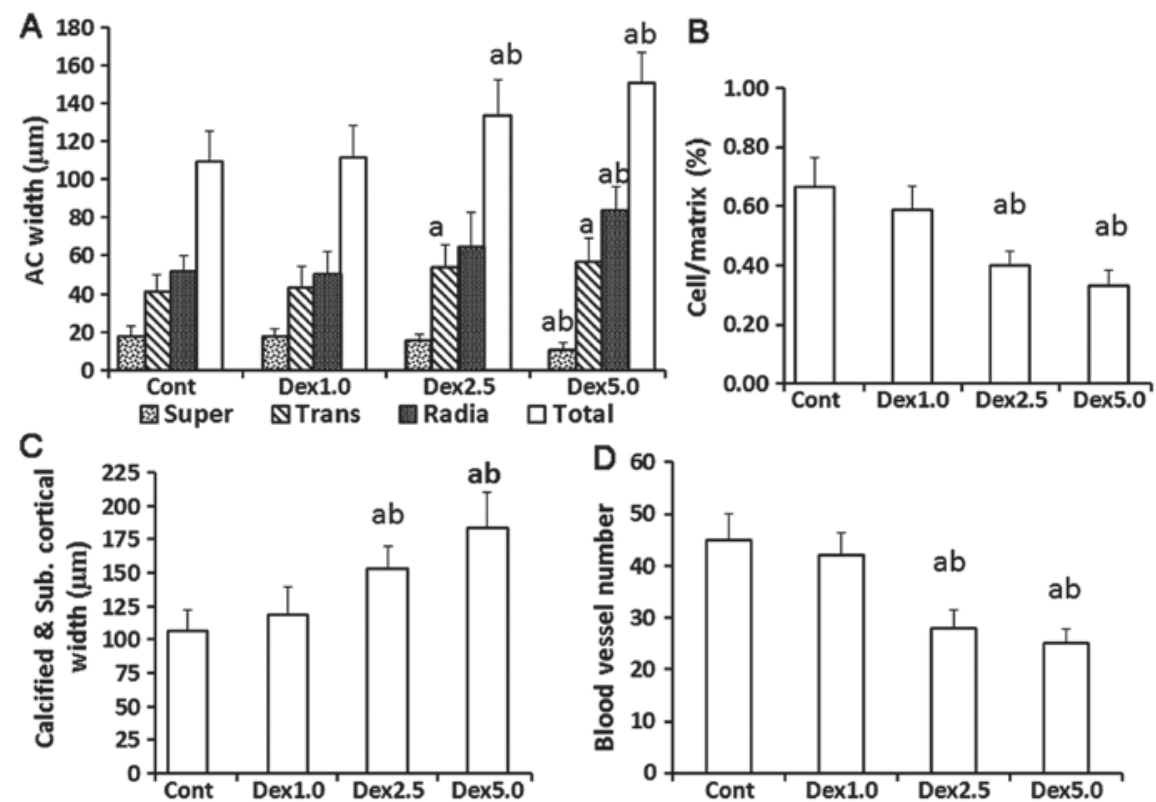

Figure 2. Parameters of the AC and subchondral zone from rats treated with vehicle or three doses of Dex. (A) Comparison of the AC width in each group. (B) Cell/matrix volume ratio of the AC zones in each group. (C) Calcified and subchondral cortical plate width changes in the subchondral zone in each group. (D) Number of blood vessels within the subchondral cortical plate in each group. ${ }^{\mathrm{a}} \mathrm{P}<0.05$ vs. Cont; ${ }^{\mathrm{b}} \mathrm{P}<0.05$ vs. Dex 1.0 . Dex, dexamethasone; Cont, control group; Dex1.0, Dex $1.0 \mathrm{mg} / \mathrm{kg}$ group; Dex2.5, Dex $2.5 \mathrm{mg} / \mathrm{kg}$ group; Dex5.0, Dex $5.0 \mathrm{mg} / \mathrm{kg}$ group; Super, superficial zone, Trans, transitional zone; Radia, radial zone; Total, total AC width; AC, articular cartilage.

presented in Table I. BV/TV, Tb.N and Tb.Th were decreased, while Tb.Sp of the trabecular epiphysis was increased in the
Dex 5.0 group compared with the control group, but Dex 1.0 and Dex 2.5 were not observed to affect these parameters. BFR was 
Table I. Histomorphometric parameters of the epiphyseal trabecular bone of the tibia in rats treated with vehicle or Dex.

\begin{tabular}{lccrr}
\hline Parameter & Cont & Dex1 0 & Dex2.5 & Dex5.0 \\
\hline BV/TV $(\%)$ & $35.65 \pm 4.41$ & $35.19 \pm 4.54$ & $34.57 \pm 3.61$ & $30.47 \pm 3.24^{\mathrm{a}, \mathrm{c}}$ \\
Tb.N $(\mathrm{N} / \mathrm{mm})$ & $3.87 \pm 0.54$ & $3.62 \pm 0.52$ & $3.35 \pm 0.59$ & $3.19 \pm 0.46^{\mathrm{a}, \mathrm{c}}$ \\
Tb.Sp $(\mu \mathrm{m})$ & $181.45 \pm 29.29$ & $184.55 \pm 22.22$ & $198.91 \pm 26.31$ & $208.95 \pm 27.70^{\mathrm{a}}$ \\
Tb.Th $(\mu \mathrm{m})$ & $102.81 \pm 7.75$ & $98.93 \pm 4.54$ & $95.67 \pm 7.84$ & $87.56 \pm 5.19^{\mathrm{a}, \mathrm{c}}$ \\
MS/BS $(\%)$ & $17.40 \pm 2.52$ & $11.24 \pm 4.52^{\mathrm{b}}$ & $7.81 \pm 2.84^{\mathrm{b}, \mathrm{c}}$ & $5.70 \pm 2.01^{\mathrm{b}, \mathrm{d}}$ \\
BFR/BS $(\mu \mathrm{m}) / \mathrm{d} * 100)$ & $19.15 \pm 2.73$ & $12.65 \pm 4.74^{\mathrm{b}}$ & $7.62 \pm 2.55^{\mathrm{b}, \mathrm{c}}$ & $5.48 \pm 2.06^{\mathrm{b}, \mathrm{d}}$ \\
BFR/TV $(\% /$ year$)$ & $48.85 \pm 8.97$ & $33.64 \pm 12.12^{\mathrm{b}}$ & $22.90 \pm 6.58^{\mathrm{b}, \mathrm{c}}$ & $15.45 \pm 4.01^{\mathrm{b}, \mathrm{d}}$ \\
Ob.S/BS & $0.87 \pm 0.12$ & $0.71 \pm 0.11$ & $0.55 \pm 0.08^{\mathrm{a}}$ & $0.32 \pm 0.05^{\mathrm{b}, \mathrm{d}}$ \\
Oc.S/BS & $0.88 \pm 0.15$ & $0.75 \pm 0.16$ & $0.65 \pm 0.17$ & $0.62 \pm 0.20^{\mathrm{a}}$ \\
Osteocyte.V/TV & $2.11 \pm 0.02$ & $3.04 \pm 0.05$ & $7.56 \pm 0.10^{\mathrm{b}, \mathrm{c}}$ & $8.45 \pm 0.14^{\mathrm{b}, \mathrm{d}}$
\end{tabular}

Data are presented as the mean \pm standard deviation. ${ }^{\mathrm{a}} \mathrm{P}<0.05,{ }^{\mathrm{b}} \mathrm{P}<0.01$ vs. Cont; ${ }^{\mathrm{c}} \mathrm{P}<0.05$, ${ }^{\mathrm{d}} \mathrm{P}<0.01$ vs. Dex 1.0 . Dex, dexamethasone; Cont, control group; Dex 1.0, Dex 1.0 mg/kg group; Dex2.5, Dex $2.5 \mathrm{mg} / \mathrm{kg}$ group; Dex5.0, Dex 5.0 mg/kg group; BS, bone surface; TV, total volume; MS, mineralizing surface; BFR, bone formation rate; BV, bone volume; Tb.N, trabecular number; Tb.Sp, trabecular spacing; Tb.Th, trabecular thickness; Ob.S, osteoblast surface; Oc.S, osteoclast surface; Osteocye.V/TV, osteocyte lacuna volume.
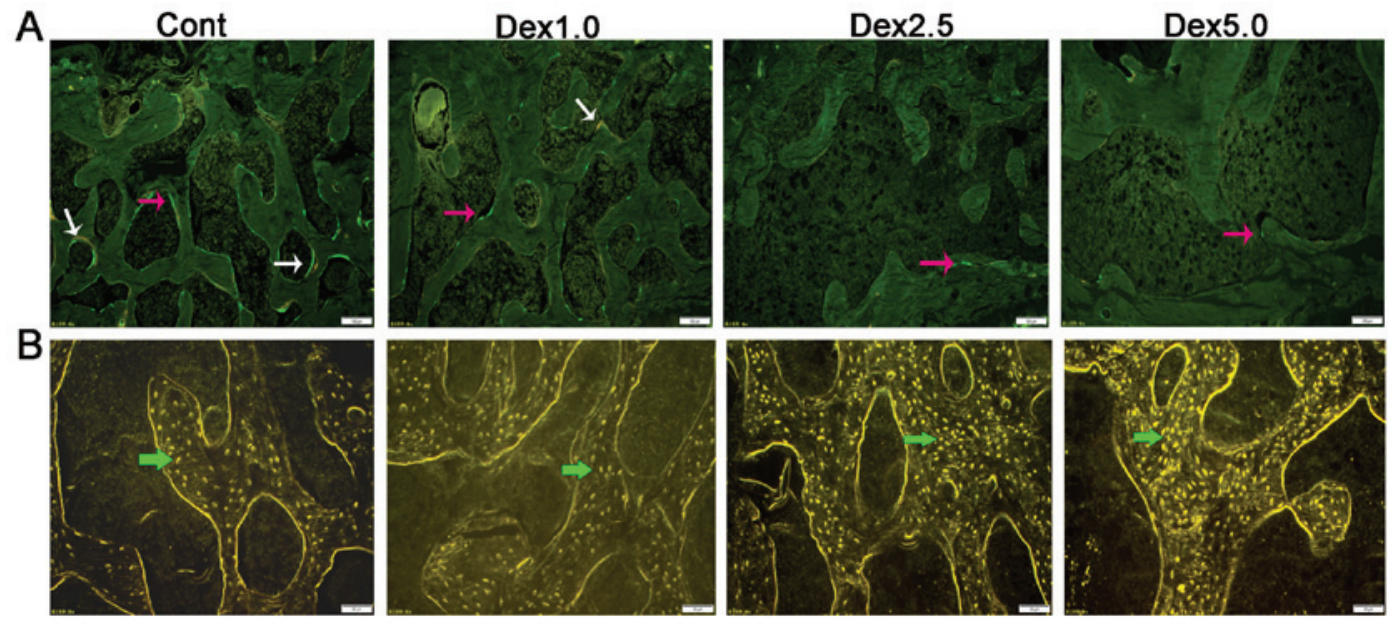

Figure 3. Microphotographs of fluorescence and autofluorescence of subchondral trabecular bone from rats treated with three doses of Dex. (A) Fluorescence images of subchondral trabecular bone. Fluorescent labeling surrounds the trabecular bone. White arrows point to double labels for tetracycline (yellow label) and calcein (green label). Pink arrows point to single calcein labels. Magnification, x10. (B) Autofluorescence image of trabecular bone. Green arrows point to osteocyte lacunae. Magnification, x20. Dex, dexamethasone; Cont, control group; Dex1.0, Dex 1.0 mg/kg group; Dex2.5, Dex 2.5 mg/kg group; Dex5.0, Dex $5.0 \mathrm{mg} / \mathrm{kg}$ group.

significantly and dose-dependently lower in all Dex groups. Ob.S/BS decreased and osteocyte lacuna volume increased in the Dex2.5 and Dex5.0 groups compared with the control group, and a greater effect was observed for Dex5.0 compared with Dex 1.0. Furthermore, Oc.S/BS was significantly lower in the Dex5.0 group compared with the control group. MS/BS and BFR were dose-dependently and significantly reduced in all Dex groups compared with the control groups. As indicated in Fig. 4, the morphology of the osteoblasts became slender, flat and inactive with irregular shaped giant osteoclasts, which appeared due to increasing doses of Dex.

\section{Discussion}

Osteoporosis and osteonecrosis of the femoral head are the two most common adverse reactions associated with glucocorticoid use (12). Currently, it is accepted that glucocorticoids directly and simultaneously influence articular cartilage as well as bone tissue, as it has been demonstrated that both structures have glucocorticoid receptors $(13,14)$. Both the articular cartilage and its adjacent bone are inevitably damaged during osteonecrosis of the femoral head. The current results indicate that Dex has a dose-dependent detrimental effect on the articular cartilage of the knee and the subchondral bone. At a given level of glucocorticoid exposure, the degree of damage to the articular cartilage was lower compared with that observed in subchondral bone and did not show obvious degeneration. In addition, the subchondral bone underwent unbalanced remodeling and osteopenia resulting from severely impeded bone formation that exceeded the reduction in bone resorption.

Articular cartilage, similar to bone, is essential for the normal functioning of joints and the skeletal system (15). 


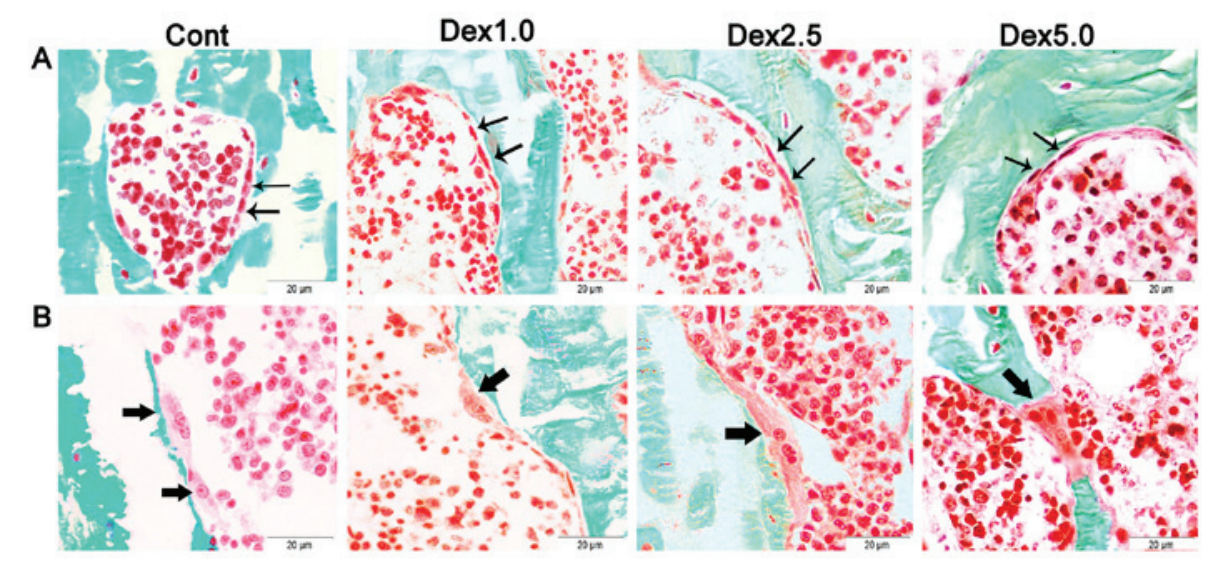

Figure 4. Osteoblast and osteoclast morphology changes in subchondral trabecular bone from rats treated with three doses of Dex. Tissues were stained with Masson Goldner trichrome bone stain. Magnification, x40. (A) Black arrows point to osteoblasts. (B) Black arrows point to osteoclasts. Dex, dexamethasone; Cont, control group; Dex1.0, Dex $1.0 \mathrm{mg} / \mathrm{kg}$ group; Dex2.5, Dex $2.5 \mathrm{mg} / \mathrm{kg}$ group; Dex5.0, Dex $5.0 \mathrm{mg} / \mathrm{kg}$ group.

The tibia articular cartilage is divided into four zones. While the superficial zone provides a gliding function and behaves as a visco-elastic and hyper-elastic material going through fast deformations during loading, the transitional and radial zones support weight and distribute stress across the cartilage to the subchondral bone $(16,17)$. The calcified cartilage zone, the thickness of which is linearly related to the degree of degeneration of the non-calcified zone, undergoes a transition that transforms the shear force into pressure and tension and then spreads it to the subchondral layer (18). Previous studies have demonstrated that excessive glucocorticoid treatment alters the cartilage chondrocyte metabolism, induces apoptosis and changes the intra-articular environment, in addition to inhibiting the synthesis of the extracellular matrix (19). However, the current study revealed that a greater thickness of the articular cartilage attributed to a greater proportion of extracellular matrix in the transitional and radial zones, as the cell/matrix volume ration in the articular cartilage was decreased. This observation implies that the increase in extracellular matrix may be a compensatory mechanism that serves to maintain joint function while redistributing stress across the cartilage $(20,21)$, rather than a direct reaction to glucocorticoid therapy. Extracellular matrix is mainly secreted by chondrocytes. As the main cells of the articular cartilage, chondrocytes with similar cell and matrix morphologies perform similar biomechanical functions. Previous results have indicated that glucocorticoids inhibit the differentiation and proliferation of chondrocytes and promote their apoptosis (22). Chondrocytes in the hypertrophic or deep zone are most vulnerable to attack as the effects of glucocorticoids highly depend on the activation and differential stage of the cells targeted in the joint (23). In the current study, the morphological changes of the radial layer chondrocytes were observed, which supported this theory. Another reasonable explanation for thicker articular cartilage is that extracellular matrix has not yet calcified, which corresponds well with the observed decrease in blood vessels in the subcostal cortical plate. Decreased angiogenesis is considered an important pathological change and an etiological factor during the development of osteonecrosis of the femoral head $(24,25)$. The reduction in nutrient supply to the calcified layer of the articular cartilage would also lead to articular cartilage degeneration.

At an identical dose of Dex, damage to the subchondral bone was greater than that observed in the articular cartilage. Furthermore, bone formation was the most sensitive indicator of bone reaction to glucocorticoid. A previous study demonstrated that the role of the subchondral bone during development of osteonecrosis of the femoral head has been underestimated, since a subchondral bone lesion is the first event that leads to the subsequent collapse of the femoral head (26). In osteonecrosis, cells of the trabecular bone, including osteoblasts, osteocytes and osteoclasts, spontaneously die, resulting in fracture and collapse of the articular surface in the femoral head (27). Both in vivo and in vitro experiments have corroborated that glucocorticoids directly inhibit osteoblast differentiation and function, and induce osteoblast apoptosis, which results in rapid and profound suppression of bone formation $(28,29)$. Glucocorticoids also directly act on osteoclasts (30). Furthermore, the altered shape of osteoclast resorption cavities profoundly reduces bone strength, while the total eroded surface area remains constant (31). Osteocytes, the most abundant bone cell type, are closely associated with systemic circulation through the lacunar-canalicular network and play a vital role in osteonecrosis of the femoral head $(7,32)$. Glucocorticoid-induced osteocyte apoptosis results in the disruption of bone vascularity and a decrease in bone hydraulic support, which causes a greater decline in bone strength compared with that due to loss of bone mass. These may be important mechanisms that underpin osteonecrosis (33). Notable, the current observations of altered morphology of osteoblasts and osteoclasts, along with inhibited bone turnover and an increase in osteocyte lacunae, are all consistent with the concepts outlined above. It was demonstrated in the current study that these developments result in unbalanced remodeling, stressed lacunar-canalicular network and a weakened bone microstructure.

The current study has certain limitations. First, Dex administration was limited to only one period of 8 weeks instead of a shorter or longer period of treatment. Second, further experiments are required to elucidate 
the mechanism of articular cartilage thickening. For instance, the expression of matrix metalloproteinase-13, type II collagen and proteoglycans in articular cartilage could be evaluated by immunohistochemistry or other molecular biology methods. Furthermore, the use of glucocorticoid antagonists, or evaluating simultaneous changes of articular cartilage and subchondral bone in both the femoral head and knee joint, could provide further insight into the mechanism by which glucocorticoid influences cartilage.

In conclusion, bone formation was inhibited at a low dose of glucocorticoid exposure, while bone resorption was reduced at higher levels of glucocorticoid treatment in rats during a period of 8 weeks. The latter effect was accompanied by an increased number of apoptotic osteocytes and resulted in unbalanced remodeling and weakened microstructure of the subchondral bone. Damage to the articular cartilage was to a lesser degree compared with in the subchondral bone, but morphological changes in chondrocytes and decreased angiogenesis were indicators of degradation of the articular cartilage.

\section{Acknowledgements}

Not applicable.

\section{Funding}

This project was funded in part by the Science and Technology Planning Project of Guangdong Province, China (grant no. 2015A030302077).

\section{Availability of data and materials}

The datasets used and/or analyzed during the current study are available from the corresponding author on reasonable request.

\section{Authors' contributions}

YC conceived the study, performed the animal experiments, analyzed data and prepared the manuscript. JZ contributed to the animal experiments and histomorphometry analyses. LH prepared the un-decalcified bone tissue sections. All authors read and approved the final manuscript for publication.

\section{Ethics approval and consent to participate}

All animal experiments were approved by the Academic Committee on the Ethics of Animal Experiments of the Guangdong Medical University, Zhanjiang, China [permit no. SYXK (GUANGDONG) A2008036].

\section{Patient consent for publication}

Not applicable.

\section{Competing interests}

The authors declare that they have no competing interests.

\section{References}

1. Whittier X and Saag KG: Glucocorticoid-induced osteoporosis. Rheum Dis Clin North Am 42: 177-189, x, 2016.

2. Cao H, Guan H, Lai Y, Qin L and Wang X: Review of various treatment options and potential therapies for osteonecrosis of the femoral head. J Orthop Translat 4: 57-70, 2015.

3. Gong LL, Fang LH, Wang HY, Peng JH, Si K, Zhu J, Han FF, Wang YH, Du GH, Pei LX and Liu LH: Genetic risk factors for glucocorticoid-induced osteonecrosis: A meta-analysis. Steroids 78: 401-408, 2013.

4. Xie XH, Wang XL, Yang HL, Zhao DW and Qin L: Steroid-associated osteonecrosis: Epidemiology, pathophysiology, animal model, prevention, and potential treatments (an overview). J Orthop Translat 3: 58-70, 2015.

5. Weinstein RS: Glucocorticoid-induced osteonecrosis. Endocrine 41: 183-190, 2012.

6. Liu R, Liu Q, Wang K, Dang X and Zhang F: Comparative analysis of gene expression profiles in normal hip human cartilage and cartilage from patients with necrosis of the femoral head. Arthritis Res Ther 18: 98, 2016.

7. Weinstein RS, Hogan EA, Borrelli MJ, Liachenko S, O'Brien CA and Manolagas SC: The pathophysiological sequence of glucocorticoid-induced osteonecrosis of the femoral head in male mice. Endocrinology 158: 3817-3831, 2017.

8. Dong YL, Zhou L, Li YL, Xiao K and Weng XS: Establishment and assessment of rat models of glucocorticoid-induced osteonecrosis. Zhongguo Yi Xue Ke Xue Yuan Xue Bao 37: 152-156, 2015.

9. Wang D, Miller SC, Liu XM, Anderson B, Wang XS and Goldring SR: Novel dexamethasone-HPMA copolymer conjugate and its potential application in treatment of rheumatoid arthritis. Arthritis Res Ther 9: R2, 2007.

10. Rentsch C, Schneiders W, Manthey S, Rentsch B and Rammelt S: Comprehensive histological evaluation of bone implants. Biomatter 4. pii: e27993, 2014.

11. Dempster DW, Compston JE, Drezner MK, Glorieux FH, Kanis JA, Malluche H, Meunier PJ, Ott SM, Recker RR and Parfitt AM: Standardized nomenclature, symbols, and units for bone histomorphometry: A 2012 update of the report of the ASBMR histomorphometry nomenclature committee. J Bone Miner Res 28: 2-17, 2013.

12. Moghadam-Kia S and Werth VP: Prevention and treatment of systemic glucocorticoid side effects. Int J Dermatol 49: 239-248, 2010.

13. Tu J, Stoner S, Fromm PD, Wang T, Chen D, Tuckermann J, Cooper MS, Seibel MJ and Zhou H: Endogenous glucocorticoid signaling in chondrocytes attenuates joint inflammation and damage. FASEB J 32: 478-487, 2018.

14. Gu Y, Zhou J, Wang Q, Fan W and Yin G: Ginsenoside Rg1 promotes osteogenic differentiation of rBMSCs and healing of rat tibial fractures through regulation of GR-dependent BMP-2/SMAD signaling. Sci Rep 6: 25282, 2016.

15. Goetzen M, Hofmann-Fliri L, Arens D, Zeiter S, Stadelmann V, Nehrbass D, Richards RG and Blauth M: Does metaphyseal cement augmentation in fracture management influence the adjacent subchondral bone and joint cartilage?: An in vivo study in sheep stifle joints. Medicine (Baltimore) 94: e414, 2015.

16. Neumann AJ, Gardner OF, Williams R, Alini M, Archer CW and Stoddart MJ: Human articular cartilage progenitor cells are responsive to mechanical stimulation and adenoviral-mediated overexpression of bone-morphogenetic protein 2. PLoS One 10: e0136229, 2015.

17. Grogan SP, Duffy SF, Pauli C, Koziol JA, Su AI, D'Lima DD and Lotz MK: Zone-specific gene expression patterns in articular cartilage. Arthritis Rheum 65: 418-428, 2013.

18. Findlay DM and Kuliwaba JS: Bone-cartilage crosstalk: A conversation for understanding osteoarthritis. Bone Res 4: 16028, 2016.

19. Liu N, Wang W, Zhao Z, Zhang T and Song Y: Autophagy in human articular chondrocytes is cytoprotective following glucocorticoid stimulation. Mol Med Rep 9: 2166-2172, 2014.

20. Tomaszewska E, Dobrowolski P and Puzio I: Morphological changes of the cartilage and bone in newborn piglets evoked by experimentally induced glucocorticoid excess during pregnancy. J Anim Physiol Anim Nutr (Berl) 97: 785-796, 2013.

21. Grogan SP, Duffy SF, Pauli C, Koziol JA, Su AI, D'Lima DD and Lotz MK: Zone-specific gene expression patterns in articular cartilage. Arthritis Rheum 65: 418-428, 2013.

22. Zhang M, Shi CY, Zhou ZL and Hou JF: Bone characteristics, histopathology, and chondrocyte apoptosis in femoral head necrosis induced by glucocorticoid in broilers. Poult Sci 96: 1609-1614, 2017. 
23. Madsen SH, Andreassen KV, Christensen ST, Karsdal MA Sverdrup FM, Bay-Jensen AC and Henriksen K: Glucocorticoids exert context-dependent effects on cells of the joint in vitro. Steroids 76: 1474-1482, 2011.

24. Zhang YL, Yin JH, Ding H, Zhang W, Zhang CQ and Gao YS: Vitamin K2 prevents glucocorticoid-induced osteonecrosis of the femoral head in rats. Int J Biol Sci 12: 347-358, 2016.

25. Weinstein RS, Wan C, Liu Q, Wang Y, Almeida M, O'Brien CA, Thostenson J, Roberson PK, Boskey AL, Clemens TL and Manolagas SC: Endogenous glucocorticoids decrease skeletal angiogenesis, vascularity, hydration, and strength in aged mice. Aging Cell 9: 147-161, 2010.

26. Wang L, Zhang L, Pan H, Peng S, Zhao X and Lu WW: Abnormal subchondral bone microstructure following steroid administration is involved in the early pathogenesis of steroid-induced osteonecrosis. Osteoporos Int 27: 153-159, 2016.

27. Briot K and Roux C: Glucocorticoid-induced osteoporosis. RMD Open 1: e000014, 2015.

28. Chen Z, Xue J, Shen T, Ba G, Yu D and Fu Q: Curcumin alleviates glucocorticoid-induced osteoporosis by protecting osteoblasts from apoptosis in vivo and in vitro. Clin Exp Pharmacol Physiol 43: 268-276, 2016.
29. Koromila T, Baniwal SK, Song YS, Martin A, Xiong J and Frenkel B: Glucocorticoids antagonize RUNX2 during osteoblast differentiation in cultures of ST2 pluripotent mesenchymal cells. J Cell Biochem 115: 27-33, 2014.

30. Teitelbaum SL: Glucocorticoids and the osteoclast. Clin Exp Rheumatol 33 (4 Suppl 92): S37-S39, 2015.

31. Vanderoost J, Søe K, Merrild DM, Delaissé JM and van Lenthe GH: Glucocorticoid-induced changes in the geometry of osteoclast resorption cavities affect trabecular bone stiffness. Calcif Tissue Int 92: 240-250, 2013.

32. Yao W, Dai W, Jiang JX and Lane NE: Glucocorticoids and osteocyte autophagy. Bone 54: 279-284, 2013.

33. Jia J, Yao W, Guan M, Dai W, Shahnazari M, Kar R, Bonewald L, Jiang JX and Lane NE: Glucocorticoid dose determines osteocyte cell fate. FASEB J 25: 3366-3376, 2011.

(c) (i) () $€$ This work is licensed under a Creative Commons cc) $\mathrm{EY}$ NG No Attribution-NonCommercial-NoDerivatives 4.0 International (CC BY-NC-ND 4.0) License. 